\title{
Micro-mixing measurement by chemical probe in homogeneous and isotropic turbulence
}

\author{
T. Lemenand ${ }^{\mathrm{a}, *}$, D. Della Valle ${ }^{\mathrm{b}}$, C. Habchi $^{\mathrm{c}}$, H. Peerhossaini ${ }^{\mathrm{d}}$ \\ a LARIS EA 7315, ISTIA, Université d'Angers, France \\ ${ }^{\mathrm{b}}$ ONIRIS, Nantes, France \\ ${ }^{\mathrm{c}}$ Notre Dame University-Louaize, Mechanical Engineering Dept., Zouk Mikael, Lebanon \\ d Univ. Paris Diderot, Université Sorbonne Paris Cité, Energy Physics Group - Astroparticle and Cosmology Laboratory, Paris, France
}

\section{H I G H L I G H T S}

- Validation of a micro-mixing model under homogeneous and isotropic turbulence (HIT).

- HIT is obtained by a system of oscillating grids placed in a vessel.

- Chemical probe measurements are carried out by the iodide/iodate reaction system.

- IEM and EDD models are tested.

- EDD model provides the more accurate quantitative characterization of micro-mixing.

\section{A R T I C L E I N F O}

\section{Article history:}

Received 7 June 2016

Received in revised form 19 October 2016

Accepted 1 December 2016

Available online 2 December 2016

\section{Keywords:}

Active grid turbulence

Chemical probe

IEM (Interaction by Exchange with the

Mean) model

EDD (Engulfment, Deformation and

Diffusion) model

Homogeneous and isotropic turbulence

Micro-mixing

LDV

\begin{abstract}
A B S T R A C T
The chemical probe is commonly used to evaluate the performance of chemical reactors. By a localized injection of chemical reagents, it is possible to measure the local micro-mixing, which is readily related to the selectivity of chemical reactions, since mixing at the molecular scale is the limiting factor for a wide range of chemical systems. The raw result of the chemical-probe method is a segregation index that allows comparison of different situations (various locations in the reactor, various Reynolds numbers, various geometries, etc.). Beyond the qualitative assessment provided by this segregation index, it is possible to obtain the intrinsic micro-mixing time by means of a micro-mixing model, describing the temporal evolution of a chemical reaction whose rate is governed by the micro-mixing. Here a key step is the choice of micro-mixing model. Several micro-mixing models available in the literature have been used in some specific cases without evaluating their appropriateness for the problem in hand. The main difficulty in this evaluation is that the real flows often do not fully satisfy the basic model assumptions, in particular the condition of homogeneous and isotropic turbulence (HIT).

The present work aims at assessing the validity of a micro-mixing model under "ideal" experimental conditions, i.e. HIT with no mean flow, to avoid the bias due to the flow gradients. The HIT is obtained here by a system of oscillating grids placed in a vessel. The chemical probe measurements carried out by the iodide/iodate reaction system are applied to the two most commonly used phenomenological models in the literature: the IEM (Interaction by Exchange with the Mean) and the EDD (Engulfment, Deformation and Diffusion) models. The benchmark for the micro-mixing models is based on comparison of the local turbulent kinetic energy (TKE) dissipation rate both drawn from the micro-mixing time by the theoretical model of Bałdyga and the reference direct experimental determination by laser Doppler velocimetry measurements. It is shown that the engulfment model EDD seems the more appropriate to analyze the chemical data and provide a quantitative characterization of micro-mixing.
\end{abstract}

(c) 2016 Elsevier B.V. All rights reserved.

\footnotetext{
* Corresponding author.

E-mail address: thierry.lemenand@univ-angers.fr (T. Lemenand).
}

\section{Introduction}

Understanding of the mechanisms that underlie turbulent mixing and convective transfer is fundamental in many technological applications - chemical, pharmaceutical, food, and cosmetic, for 
example [1]. Indeed, the selectivity of the fast chemical reactions depends strongly on the way in which the reagents are mixed on a molecular scale [2]. This mechanism is called micro-mixing, the ultimate scale of mixing by the flow before molecular diffusion comes to action. The kinetic of micro-mixing is a key parameter for the selectivity of the chemical reactions: micro-mixing must be "faster" than the chemical reaction to let the intrinsic reaction rate be effective [3].

Three parallel mechanisms can be distinguished in turbulentmode mixing: macro-mixing, meso-mixing, and micro-mixing [4]. Macro-mixing takes place at the mixer scale and represents the fluid dispersion by the mean velocity fields. A fluid in a mixer disperses under the effect of the mean flow and is finally distributed in the whole mixer volume. This stage of mixing is influenced by longitudinal or transverse swirls that contribute to convective mass transfer and macro-mixing is characterized by the residence time distribution (RTD). In laminar flow macromixing can be improved by geometries that induce a chaotic advection, which makes the flow irregular $[5,6]$.

In the presence of velocity fluctuations with no mean value, meso-mixing is related to the "random-path" turbulent agitation of fluid particles, analogous to a Brownian macroscopic motion. Nevertheless, it is governed not by a Boltzman statistics, but by a mechanism of scale reduction by the energy cascade in the inertial-convective subrange of the turbulent spectrum - wave numbers ranging between the integral and the Kolmogorov scale $\eta$ (see Fig. 1). Thus, meso-mixing is a homogenization process by advection due to velocity fluctuations. It can be characterized by the TKE $k$ (namely turbulent kinetic energy), a turbulent diffusion concept, or a dominant component of the Reynolds tensor $\overline{u_{i} u_{j}}$. In laminar flow, the meso-mixing - on intermediate scales - is related to the possible "mesoscopic" fractal structures of the flow [7].

Micro-mixing is the ultimate mixing scale in the flow; it takes place in the viscous-convective subrange, defined by $\kappa_{\eta}<\kappa<\kappa_{B}$ where $\kappa$ is the wave number, $\kappa_{B}$ is the wave number on the Batchelor scale, especially for high-Schmidt-number fluids (liquid phase) when these scales are very different. In this range, turbulent fluctuation has already vanished and mixing is due to laminar stretching proportional to $(\varepsilon / v)^{1 / 2}$, with $\varepsilon$ the dissipation rate of the turbulent kinetic energy and $v$ the kinematic viscosity, that reduces the species clusters on the molecular diffusion scale [8-10]. At the Batchelor scale, the molecular diffusion quickly dissipates the concentration variance, permitting homogenization of the reagents at molecular scale. The limiting stretching mechanism is related to engulfment in the small vortices near the Kolmogorov scale [4]. Consequently, the micro-mixing depends on the dissipation rate of the turbulent kinetic energy $\varepsilon$, or equivalently on the Kolmogorov scale $\eta$.

Actually, if the reaction kinetics is faster than the micro-mixing, the reaction yield is reduced by an insufficient reagent feeding, and the production of by-products is favored; that is why micro-mixing is straightforwardly related to reaction selectivity. In some cases, micro-mixing also affects product quality: for instance, precipitation reaction [11-13] and polymerization reactions [14].

Micro-mixing is responsible for the global performance of multifunctional heat exchanger reactors (MHER) when the mixing mechanisms on the larger scales - macro and meso-mixing are not limiting.

Several methods have been reported for characterizing micromixing [15]; functional methods fall into three main categories. The first is based on color modification in a solution containing a reacting system directly injected in the flow, such as a $\mathrm{pH}$ indicator of an acid-base solution. To obtain quantitative information on mixing efficiency, mixing time is estimated by the ratio of the length of the color variation to the mean flow velocity [16]. The second method involves a spatiotemporal recording of the concentrations to determine a coefficient of variance (COV) and then the segregation index [17]. The third technique, developed by Villermaux [18] and reviewed by Fournier et al. [19] and Guichardon et al. [20,21], entails competitive-consecutive or competitiveparallel reactions that are based on the chemical result of the local injection of a reagent in stoichiometric deficit in the main flow. The main reaction is quasi-instantaneous with a characteristic time $t_{\mathrm{r} 1}$; the side reaction is slower with characteristic time $t_{\mathrm{r} 2}$ that must be close to the mixing time, so that micro-mixing is limiting for this reaction.

Among these methods, the last-named has been successfully used to evaluate micro-mixing in batch and open-loop reactors with different chemical systems; most of them are consecutivecompeting reactions, such as the diazo-coupling test [22], while others are competitive-parallel reactions, such as the iodide/iodate chemical test $[19,20,23,24]$, the method used in the present study.

The principle of the chemical probe is to measure the proportion of the by-products produced due to the secondary slower

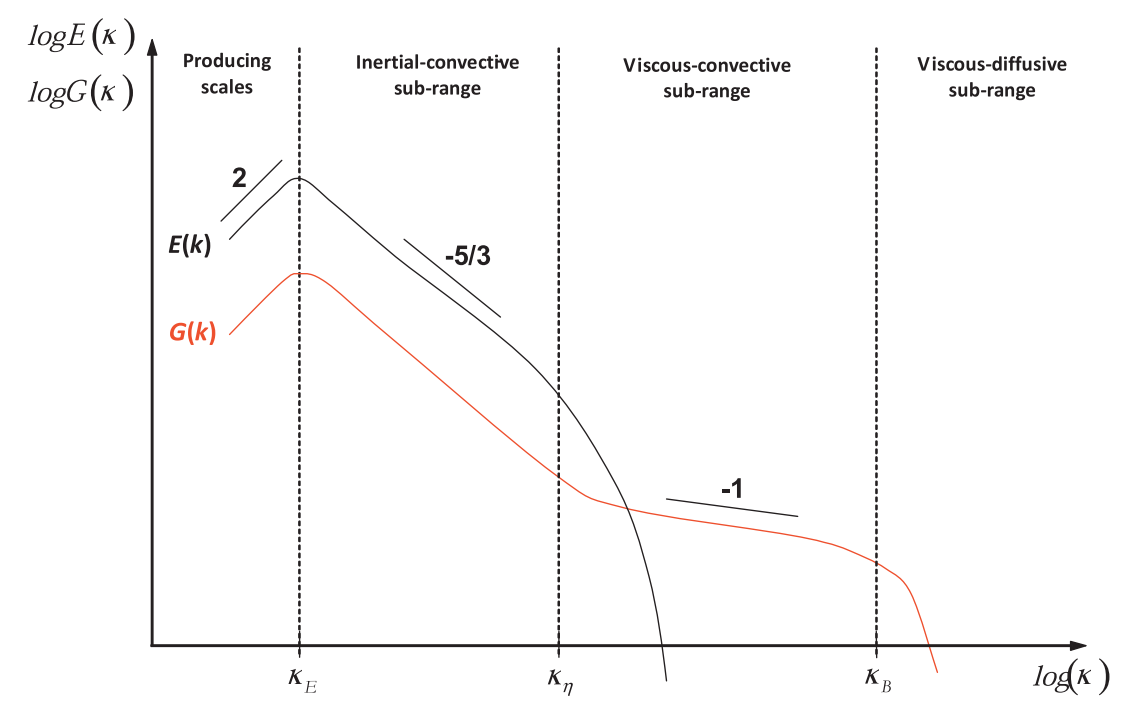

Fig. 1. Spectra of turbulent kinetic energy $E(\kappa)$ and scalar $G(\kappa)$ versus wave number $\kappa$ (for Schmidt numbers $\gg 1$ ). 
reaction, which is a signature of imperfect mixing and insufficient renewal of the primary-reaction reagents: it leads to the definition of a segregation index $X_{S}$ in the range [0-1]. In perfect mixing, the common reagent is totally consumed by the quasi-instantaneous reaction so that $X_{S}=0$. In total segregation, an overconcentrated zone of the injected common reagent occurs and the slower reaction can react with this common reagent, so that $X_{S}=1$. To obtain this value accurately, a series of conditions and hypotheses are necessary: i) the reaction is fully achieved locally, ii) the measurement is objective, i.e. there is no interference with the injection hydrodynamics, and iii) the need to target values of $X_{S}$ "far" from 0 and 1 requires adapting concentrations to the flow turbulence level [25].

Nevertheless, $X_{S}$ always depends on the concentrations and the protocol used, and hence can offer a comparative criterion only in similar situations. The intrinsic parameter characterizing the absolute magnitude of the reaction apparent kinetics is the micromixing time $t_{m}$ (the inverse of a mixing rate), which can be considered the key parameter of chemical reactor performance for rapid reactions. The mixing time can be related to the segregation index by using physical models [26-29].

The relevant micro-mixing model for deducing $t_{m}$ from $X_{S}$ measurements is the focus of the present work. Micro-mixing models are built on the local consumption of the species, and transfers by diffusion or by convection are basically not taken into account. At this level, rigorous application of the micro-mixing models needs to be handled in a batch, and moreover in zero-gradient conditions, that is homogeneous.

The micro-mixing models in the literature for large-Schmidtnumber fluids $\left(S_{c} \approx 1000\right)$ are in general of two kinds: deterministic models, in which the concentrations are computed by the species concentrations at a given rate [4], and stochastic models, in which the concentration evolution is modeled by an Eulerian PDF (probability density function) approach (from given statistics of the velocity fluctuations) associated with a Monte-Carlo method [30]. It can be noticed that a PDF transform of the mass balance equation can also be useful in treating fast reactions in CFD (computational fluid dynamics) models [8] for its ability to handle the discrepancy between momentum and mass transfer time-scales, and also because in this formulation the chemical diffusive term does not need a closure hypothesis.

This study is aimed to test two deterministic models: the IEM (Interaction by Exchange with the Mean) and EDD (Engulfment, Deformation and Diffusion) models. Then, the major issue in validating a micro-mixing model is to compare the micro-mixing time obtained from chemical reaction measurements (chemical probe) with the measurements obtained by a model-independent (direct) method. The way to work this out is to use Baldyga's model, which relates $t_{m}$ to the turbulent kinetic energy (TKE) dissipation rate $\varepsilon$, established for isotropic and homogeneous turbulence from the analysis of the more energetic Kolmogorov vortices [4]:

$t_{m}=17.24\left(\frac{v}{\varepsilon}\right)^{1 / 2}$

The chemical measurements processed with the micro-mixing models lead to $\varepsilon$ values that can then be compared with those obtained from laser Doppler velocimetry (LDV) measurements in the same vessel where the homogeneous isotropic turbulence (HIT) is generated by two vertically oscillating grids. This type of turbulence generator is frequently used for studying mixing and chemical reactions [31].

The paper is organized as follows. Section 1 presents the description and validation of the HIT generation system. The purpose is at first to verify the homogeneity and the isotropy of turbulence by LDV measurements, at least in a significant zone of the vessel core, i.e. in the central part where the injection is carried out. The experimental TKE dissipation rate is also determined at this stage. Section 2 presents the chemical probe data leading to the segregation index, in various conditions of stroke and frequency of the grid oscillations. Section 3 provides a description of micro-mixing models and discusses their application to the data, by comparison between $\varepsilon$ resulted from micro-mixing time $t_{m}$, and the reference value obtained by optical measurement. Concluding remarks are given in Section 4.

\section{Homogeneous and isotropic turbulence}

\subsection{Active grid apparatus}

Two general types of devices are used to generate (approximately) the ideal case of homogeneous and isotropic turbulence: the flow behind a grid in a channel [32] or the oscillation of one or more grids in a tank [33]. The first experimental system conducted in wind and water tunnels had a mean flow component across the grid. However, turbulence decays rapidly, and thus their practical utility is limited. An alternative is vibrating grid turbulence generated in a water tank by a planar grid vibrating vertically to the observation plane. This flow has commonly been used to investigate entrainment and mixing mechanisms. A vertically oscillating grid can generate nearly zero-mean-velocity turbulence over a wide area with turbulence characteristics (velocity and length scales) determined by the grid geometry, the stroke $S$ and frequency $f$ of oscillation, and the vertical distance $z$ from the grid.

The experimental system designed and constructed in this work is shown in Fig. 2. The apparatus is a transparent rectangular glass tank of inner dimensions $130 \times 130 \times 330 \mathrm{~mm}$ allowing optical measurements such as LDV and PIV, including a pair of horizontally oriented grids and an associated vertically oscillated driving device.

Each grid is composed of ten square-cross-section bars arranged in a square array with $M / d=5$, where $M$ is the grid mesh size and $d$ the bar width. In order to minimize tank wall effects (reflection by the walls of the large flow structures produced by the grids), the grid outer extremities are equipped with half-meshes [34], as shown in Fig. 2(a). The grid dimensions define its compactness or density $\delta$ :

$\delta=\left(2 \frac{M}{d}-1\right)\left(\frac{M}{d}\right)^{-2}$

The grids are manufactured by laser cutting in a 5-mm-thick stainless steel plate. At each tank corner, four vertical rods allow grid actuation and guidance. The grids are fixed on two threaded rods by means of nuts and two copper spacers corresponding to the height $H$. The two other rods are smooth and are fixed at the bottom of the tank. They allow guidance of the grids by a sliding sleeve of minimal friction. A transmission chain transforms the rotational movement of the electric motor into translation movement thanks to a "rod-crank" system, shown in Fig. 2(c). The ratio of the rod length to the crank radius is at least twenty in order to ensure satisfactory sinusoidal oscillation. The oscillation frequency can be adjusted by the variable speed of the electric motor. The stroke is regulated by modifying the rod eccentricity. The dimensions of the tank and the grids and other technical data are presented in Table 1.

The grid compactness defined by Eq. (2) is 36\%. It is lower than the stability threshold of the grid wake which is $40 \%$, in order to avoid strong non-homogeneity and secondary circulation flows [34]. 

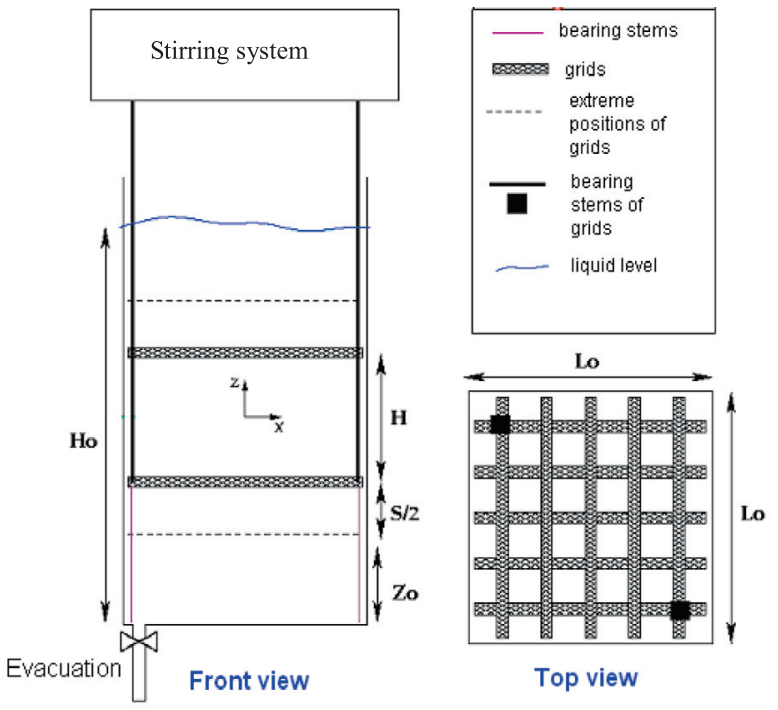

Top view

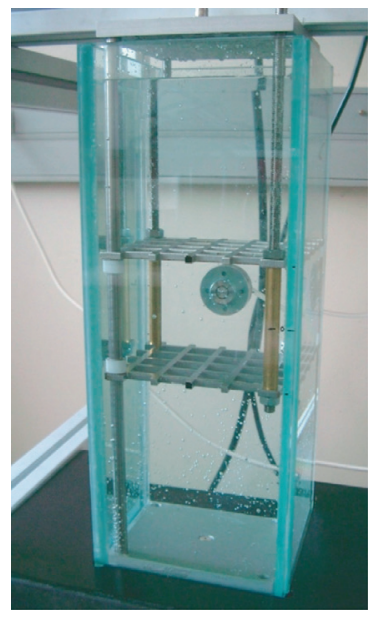

(b) (a)

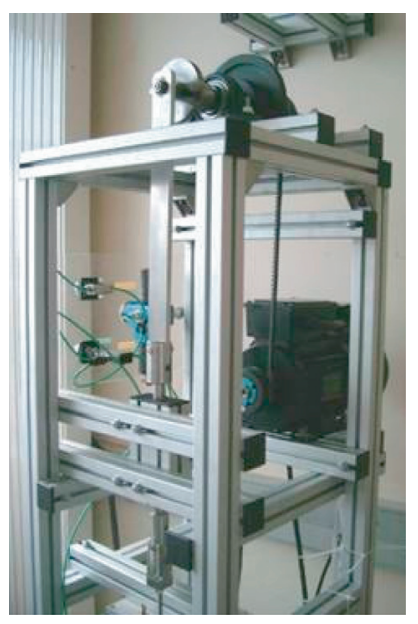

(c)

Fig. 2. Oscillating grid system in a tank: (a) principle of tank stirring by oscillating grids, (b) view of the grid pair, (c) motorization and transmission located above the tank.

Table 1

Tank dimensions, grid dimensions and technical data.

\begin{tabular}{lll}
\hline Tank dimensions & Height & $H_{0}=330 \mathrm{~mm}$ \\
& Width & $L_{0}=130 \mathrm{~mm}$ \\
Grids dimensions & Mesh size & $M=25 \mathrm{~mm}$ \\
& Bars width & $d=5 \mathrm{~mm}$ \\
& Grid compactness & $\delta=36 \%$ \\
Technical data & Oscillation frequency & $f[2-10] \mathrm{Hz}$ \\
& Gap between the grids & $H[80-140] \mathrm{mm}$ \\
& Grid stroke & $S[20-40] \mathrm{mm}$ \\
\hline
\end{tabular}

\subsection{Turbulence measurements}

\subsubsection{Experimental procedure}

The turbulence intensity generated by the system depends on three main parameters: the oscillation frequency $f$, the distance between the grids $H$ and the grids stroke $S$. A compromise among these parameters must be found in order to preserve as much as

possible the isotropy and homogeneity of turbulence (large gaps, weak strokes and appropriate frequencies).

The velocity field is measured by LDV on the three axes of the orthonormal frame at the center point of the vessel (Fig. 3). The measurement points are separated $5 \mathrm{~mm}$ one the other, up to a distance of $20 \mathrm{~mm}$ on each direction. Item 0 corresponds to the velocity measurement at the center of the symmetry plane.

The LDV measurements in the three planes provide the mean and fluctuating components of the velocity in the horizontal and vertical directions, as well as the six Reynolds stress components. Reproducibility is evaluated at $5 \%$ standard error, evaluated by three successive measurements on the same run, where the gap and stroke are re-initialized between each test, by adjustment of the eccentricity and disassembly of the grids.

\subsubsection{Correlations for oscillating grid turbulence}

With an oscillating grid in a tank, the evolution on the $z$ axis of the standard deviation (denoted by "rms", the root mean square) of the turbulent velocity components in the horizontal plane, $u_{r m s}$ and $v_{r m s}$, at a distance $z$ measured from the midplane of the oscillating grid, is given by:

$$
u_{r m s}=v_{r m s}=C_{1} S^{3 / 2} M^{1 / 2} f z^{-1}
$$

with $C_{1}$ a numerical constant varying from 0.20 to 0.50 in different works [31,35-38], for mesh grids $M$ in the range [30-80] $\mathrm{mm}$ and strokes $S$ in the range [8-45] $\mathrm{mm}$. The grids are in the horizontal plane $(x, y)$ and oscillate in the vertical direction $z$. The two velocity components $u_{r m s}$ and $v_{r m s}$ are equal by symmetry. Sufficiently far from the grids (at approximately seven times the mesh size $M$ ), the power of $z$ in Eq. (3) is almost -1.5 .

In the case of two oscillating grids, Villermaux et al. [37] proposed:

$u_{r m s}=v_{r m s}=2^{1 / 3} C_{1} S^{3 / 2} M^{1 / 2} f z^{-1}$

A slight anisotropy is generally noted in grid turbulence [35]:

$\frac{W_{r m s}}{u_{r m s}} \sim 1.20$ and $\frac{W_{r m s}}{v_{r m s}} \sim 1.20$

with $w_{r m s}$ the rms velocity component in the vertical direction.

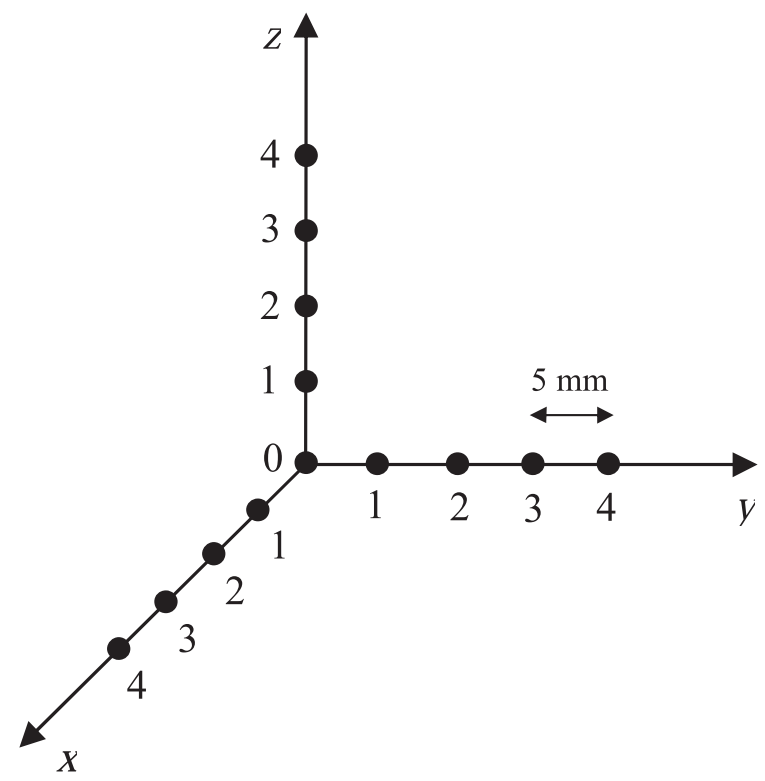

Fig. 3. Measurement point locations by LDV. Point 0 is the vessel center. 


\subsection{Homogeneity and isotropy in the vessel core}

\subsection{1. $L D V$ measurements in the vessel center and vicinity}

Temporal signals of the longitudinal velocity fluctuations are recorded using LDV at the locations marked in Fig. 3. The spectra are presented in pre-multiplied format, i.e. based on the power spectral density multiplied by the frequency plotted versus frequency on a semi-log scale. The turbulent kinetic energy (TKE) $k$ is hence visualized by the area below the curve $[39,40]$. As an example, the pre-multiplied spectra of velocity fluctuations for a frequency of $6 \mathrm{~Hz}$, a gap of $120 \mathrm{~mm}$, and a stroke of $40 \mathrm{~mm}$, for the two velocity components $u$ and $w$ on the $x$ and $z$ coordinates are shown in Fig. 4 (the horizontal velocity component $v$ is not shown because it is identical to $u$ for symmetry reasons).

A satisfactory homogeneity (spatial invariance) is observed on the $(x, y)$ plane since (as shown in Fig. 4(a) and Fig. 4(c)) all profiles merge to a single curve. An energy peak appears at grid oscillation frequency $6 \mathrm{~Hz}$, which corresponds to the flow structures released by the grid; however, this remains a weak contribution to the global kinetic energy. The homogeneity on the vertical axis can be observed in Fig. 4(b) and Fig. 4(d) and is valid up to $z=10 \mathrm{~mm}$. This implies that a zone of $10 \mathrm{~mm}$ around the central point 0 can be considered homogeneous.

The root mean square values of the turbulent velocity components are in fair agreement with the theoretical model in Eq. (4)

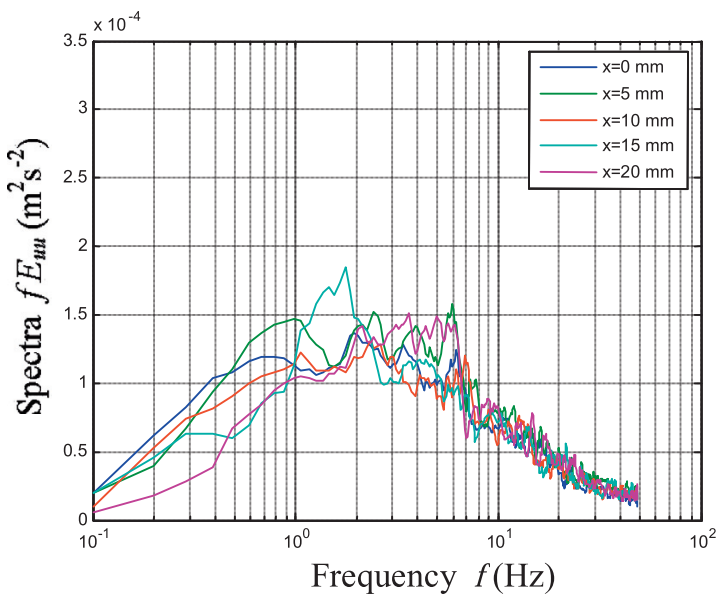

(a) $u$ component along $X$

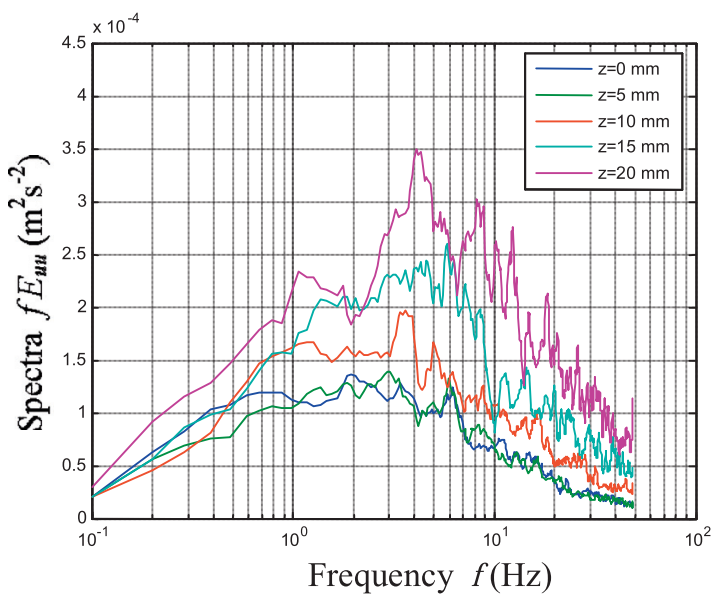

(b) $u$ component along $Z$ as shown in Fig. 5; the deviation between theory and measurements is less than $6 \%$. In this case, the constant $C_{1}=0.233$.

\subsubsection{Isotropy}

The ratio of fluctuating velocity components for the vertical and horizontal directions $w_{r m s} / u_{r m s}$ shown in Fig. 6 characterizes the isotropy provided by the active grid system at the center of the vessel, and lies at around $1.30 \pm 6 \%$, of the same order found in the literature (Eq. (5)). The isotropy is better for the smaller strokes $(S=20 \mathrm{~mm})$ and the larger distances between the grids $(H=100$ and $120 \mathrm{~mm})$, but no general law seems to emerge. For example, the isotropy is excellent $\left(w_{r m s} / u_{r m s} \approx 1.00\right)$ for a frequency $f=4 \mathrm{~Hz}$, a height of $H=100 \mathrm{~mm}$, and a stroke $S=20 \mathrm{~mm}$, and also for $f=8 \mathrm{~Hz}, H=120 \mathrm{~mm}, S=20 \mathrm{~mm}$.

\subsubsection{Homogeneity}

The main advantage of using two active grids is that the central point is a zero-gradient location for symmetry reasons [37]. The issue is the robustness of this feature in the vicinity of the point 0 ; that is, to ensure a reliable 0 -dimensional situation regardless of any inaccuracy in the central location. The horizontal and vertical fluctuating velocities $u$ and $w$ appear to be quite constant in the horizontal plane $(x, y)$, with a measurement error of $6 \%$. Fig. 7 and Fig. 8 show the dimensionless group $k^{1 / 2} / f S$ calculated based on the

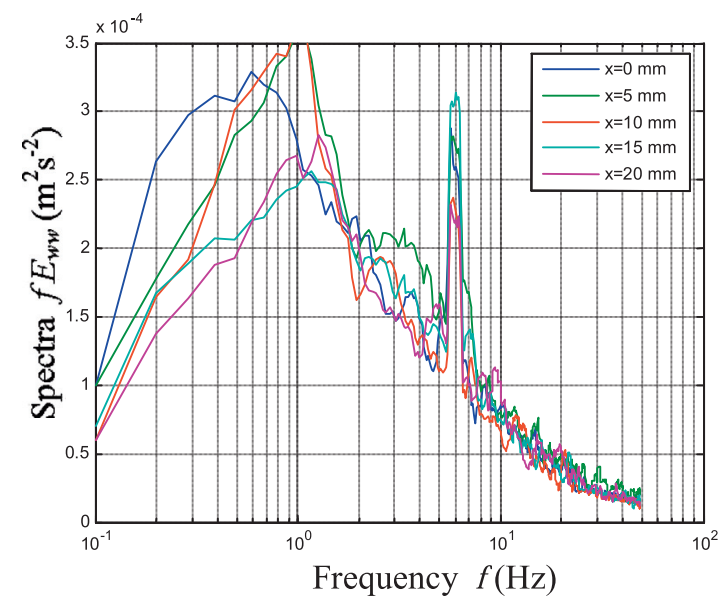

(c) $W$ component along $X$

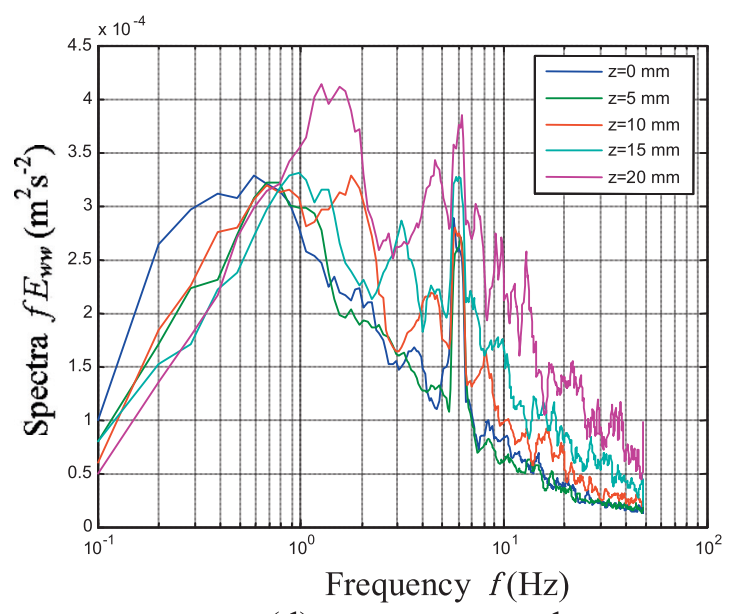

(d) $W$ component along $Z$

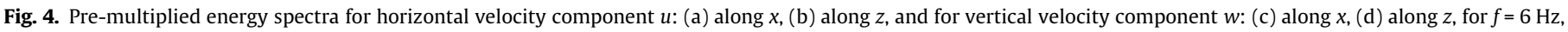
$H=120 \mathrm{~mm}, S=40 \mathrm{~mm}$. 


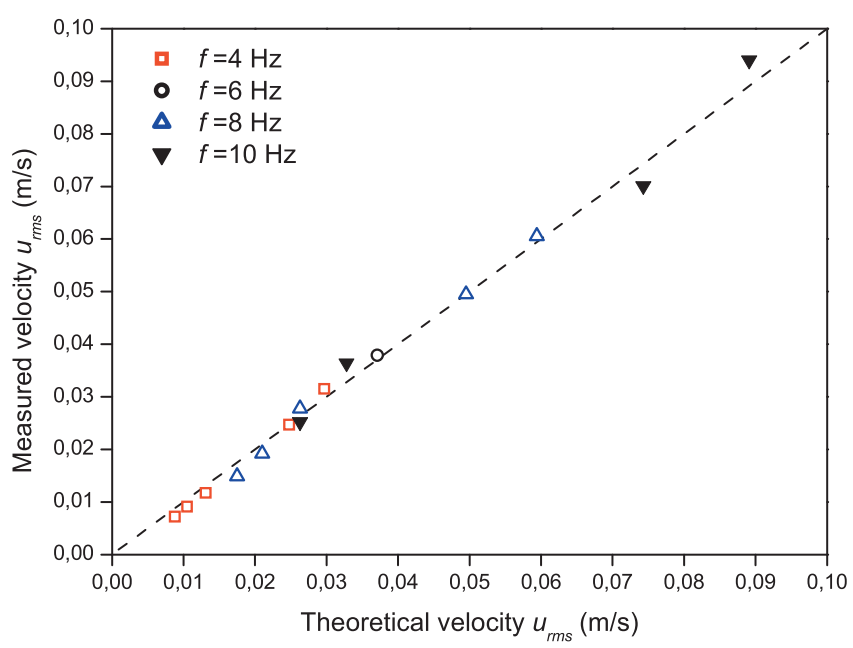

Fig. 5. Measured horizontal fluctuating velocities at center of oscillating grids (point 0 ) versus theoretical velocity of Eq. (4).

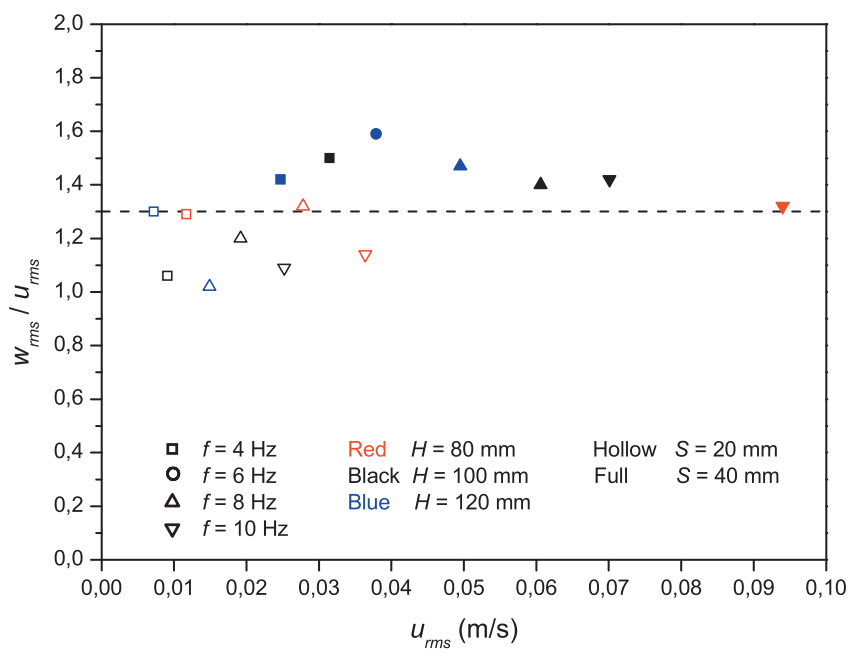

Fig. 6. Isotropy at center of oscillating grids (point 0 ).

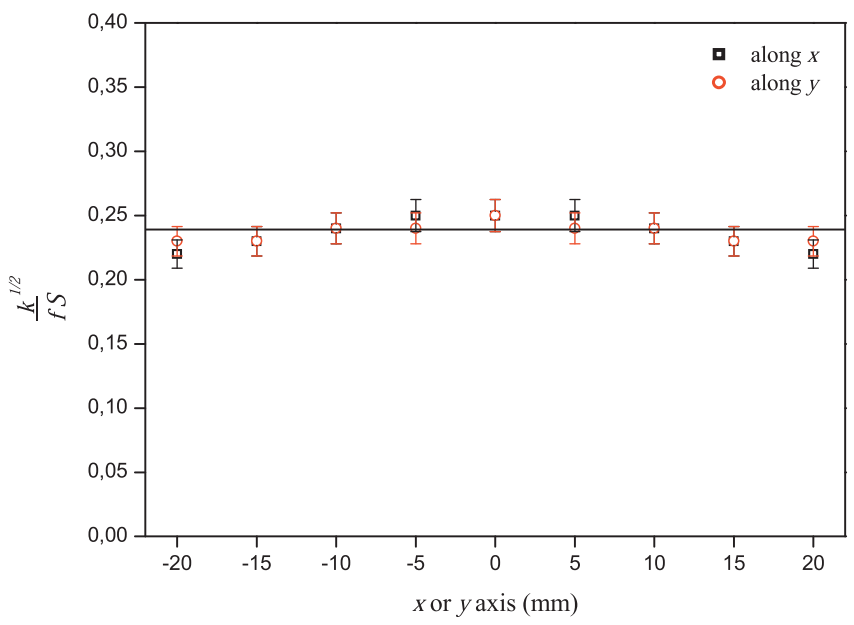

Fig. 7. Homogeneity in horizontal plane ( $\mathrm{x}$-axis and $\mathrm{y}$-axis).

TKE $k$ respectively along the $x / y$ axis and the $z$ axis, where the TKE $k$ is defined by:

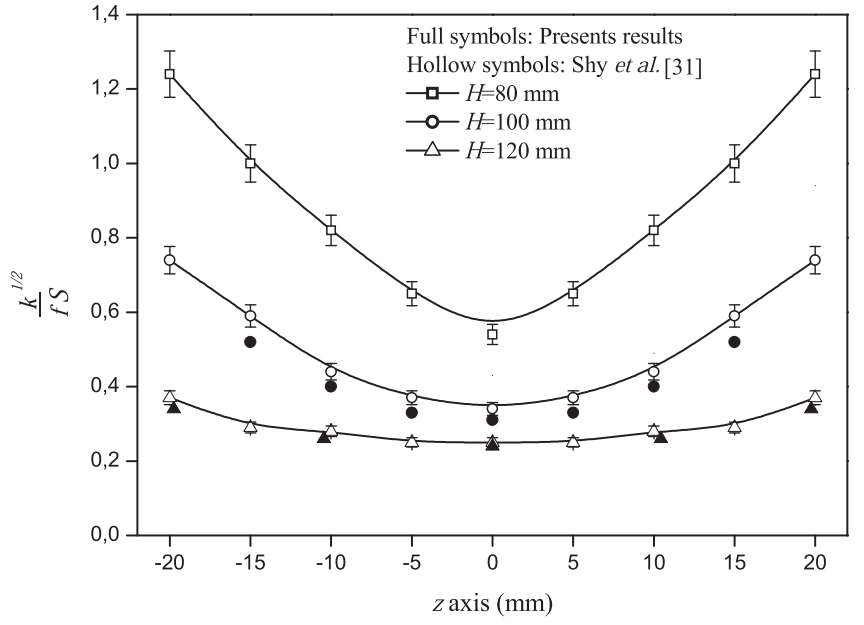

Fig. 8. Homogeneity on vertical z-axis.

$k=\frac{1}{2}\left(2 u_{r m s}^{2}+w_{r m s}^{2}\right)$

The variable $k^{1 / 2} / f S$ is quite constant along the $x$ and $y$ axes over the zone of $20 \mathrm{~mm}$ around the central point. Fig. 7 confirms the satisfactory homogeneity of the turbulent field on the whole measurement volume $20 \mathrm{~mm}$ around the central point in the horizontal plane. Fig. 8 shows that the limit of the 0 -D zone size is primarily in the $z$-axis because of the steep decay of $w$ in the $z$ direction, so that the measurement location should stay within a $10-15 \mathrm{~mm}$ distance for the grid gaps of 100 and $120 \mathrm{~mm}$, but only $5 \mathrm{~mm}$ for $H=80 \mathrm{~mm}$. The results of this work are confirmed by comparison with the measurements of Shy et al. [31], with differences less than $9 \%$ in average.

Nevertheless, all chemical probe measurements are carried out at the central point, at $x=y=z=0$, where the homogeneity is ensured regardless of any eventual slight inaccuracy in measurement location.

\subsection{Derived quantities from the turbulent field}

\subsubsection{Temporal and spatial integral scales}

The temporal integral scale $\tau$ is obtained by integration of the velocity autocorrelation function using LDV measurement data. For each operating parameter set $(f, H$ and $S)$ these scales are the average of the values measured on the horizontal coordinates $(x, y)$ (since the LDV system used allows velocity measurements on only two axes). The integral time-scale can be evaluated from the temporal correlation function, which requires velocity measurements with only a single probe. Measurements must be performed at sufficiently short time intervals to detect highfrequency fluctuations. Laser Doppler velocimetry allows such fast measurements by optimizing seeding and optical adjustments. However, since LDV measurements are not performed at constant time intervals, the data must be re-sampled according to the method suggested by Host-Madsen and Caspersen [41].

Then the turbulent energy dissipation rate can be computed from the following expression, using Batchelor's model [10] based on dimensional analysis:

$\varepsilon=A \frac{u_{r m s}^{3}}{\Lambda}$

where $A$ is a constant around unity [42], taken equal to one in this study, and $u_{r m s}$ is the velocity fluctuation intensity on the first axis as mentioned earlier. The longitudinal integral length scale $\Lambda$ is defined by: 
$\Lambda=\int_{0}^{\infty} \frac{\overline{u^{\prime}(x) u^{\prime}(x+r)}}{\overline{u^{\prime 2}(x)}} d r$

Here $u^{\prime}$ refers to the instantaneous fluctuating velocity. The spatial autocorrelation, which is not accessible by LDV measurement, is deduced from the temporal macro scale, determined by the temporal autocorrelation:

$\tau=\int_{0}^{\infty} \frac{\overline{u^{\prime}(t) u^{\prime}(t+T)}}{\overline{u^{\prime 2}(t)}} d T$

with the Taylor assumption of frozen turbulence:

$\Lambda=u_{r m s} \tau$

Values of $\Lambda$ reported in Fig. 9 are consistent with the orders of magnitude quoted in similar flow [31]. Values of $\Lambda$ are represented in comparison with the Taylor micro-scale $\lambda$ versus the Reynolds number based on the Taylor scale $R e_{\lambda}$, which is commonly used to characterize grid turbulence. The ratio $\lambda / u_{r m s}$ characterizes the time-scale of the small eddies. For homogenous and isotropic turbulence:

$\lambda=\left(\frac{30 v u_{r m s}^{2}}{\varepsilon}\right)^{1 / 2}$

and

$\operatorname{Re}_{\lambda}=\frac{u_{r m s} \lambda}{v}$

In the range of parameters of Reynolds number $R e_{\lambda}[15-80]$, the integral scale $\Lambda$ increases with $\operatorname{Re}_{\lambda}$ while the Taylor micro-scale $\lambda$ decreases, indicating the extension of the inertial range.

\subsubsection{TKE dissipation rate}

Two models to estimate the TKE dissipation rate $\varepsilon$, valid for an oscillating grid, are used. The first, due to Kit et al. [36], is suggested for an oscillating grid at frequencies $2-4 \mathrm{~Hz}$, a mesh size of $80 \mathrm{~mm}$, and a stroke of $32 \mathrm{~mm}$, in a vessel of $450 \mathrm{~mm}$. It should be noted that the fluctuating velocity used in this paper is the vertical velocity component $w_{\text {rms }}$ :

$\varepsilon=0.75 \frac{w_{r m s}^{3}}{\Lambda}$

The second model was proposed by Bache et al. [43] with an empirical correlation based on measurement of the power injected in the fluid by the grid, in the case of a fine mesh $(10 \mathrm{~mm})$ and high frequencies $(15-40 \mathrm{~Hz})$ :

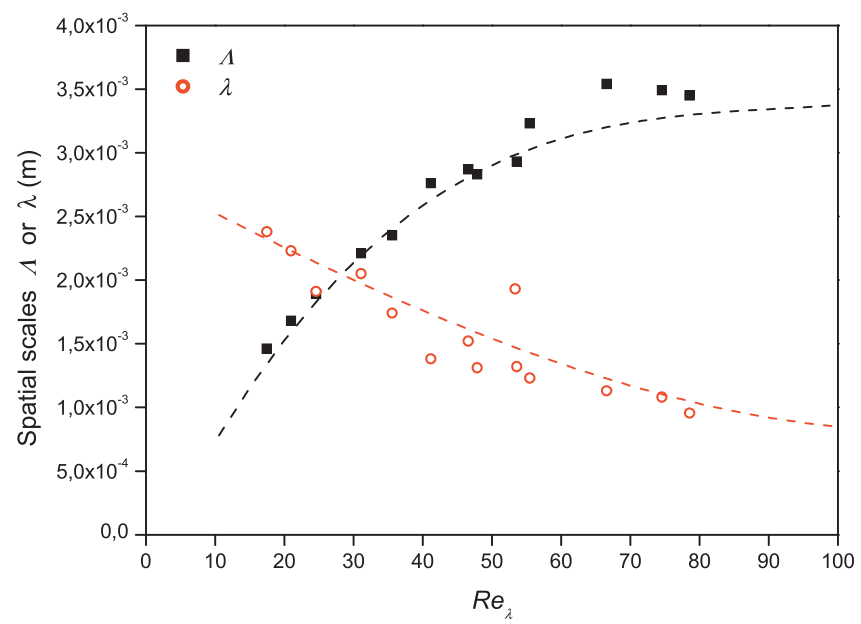

Fig. 9. Spatial integral scale $\Lambda$ and Taylor micro-scale $\lambda$. $\varepsilon=2.40 \frac{u_{r m s}^{2}}{\tau}$

The TKE dissipation rates $\varepsilon$ are plotted in Fig. 10 as a function of the TKE $k$. Values obtained by the two above methods are also plotted on the same figure; they are in relatively good agreement with the order of magnitude of the present measurements, particularly for high TKE levels. The TKE dissipation rates measured in this study appear to be underestimated compared to the literature, by a factor of 1.50 compared to the results of Kit et al. [36] and 1.94 compared to those of Bache et al. [43]. This may be due to the value of the constant $A$ in Eq. (7) (taken equal to 1 in this study), which is not an absolute constant: the constant can be greater than one, especially when turbulence is relatively weak [42]; for instance, Mohand Kaci et al. $[44,45]$ reported $A=1.85$ in a tube equipped with vortex generators at the wall.

\section{Chemical probe measurements}

\subsection{Principle of the chemical probe - iodure/iodate system}

An increasing literature proposes the use of chemical reactions for local measurement of micro-mixing in a turbulent flow [4648]. The chemical probe is generally based on the measurement of the $S$ species of the secondary reaction in a parallelcompetitive system:

$A+B \rightarrow R$

$C+B \rightarrow S$

For this, the reaction (15) is fast compared to the reaction (16), and the characteristic time $t_{r 2}$ of reaction (16) must be of the same order of magnitude as that of the micro-mixing $t_{m}$ we want to measure (the "local" mixing must be limiting). Reagents $A$ and $C$ are previously diluted in the main flow in stoichiometric excess. A small quantity of reagent $B$ (in stoichiometric deficit) is injected locally into the flow. The reaction rate is hence governed by the micro-mixing, as shown in Fig. 11. The composition of the chemicals at the exit determines the segregation index $X_{S}$, which is the ratio between the quantity of $B$ transformed into $S$ and the initial quantity of $B$.

Previous work of Fournier et al. [19] and Guichardon and Falk [20] have detailed an experimental procedure for using the

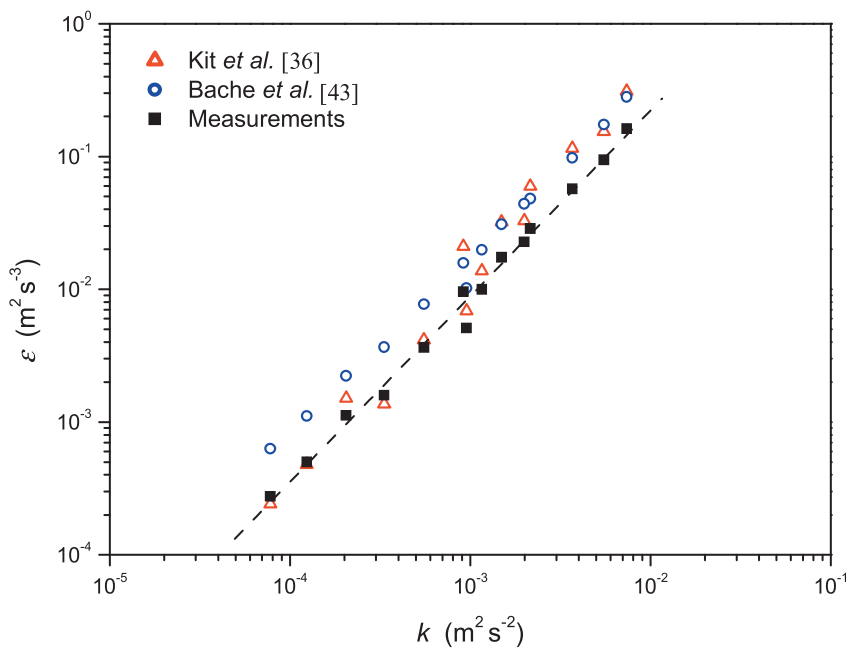

Fig. 10. TKE dissipation rate versus TKE in grid turbulence measured at point 0 comparison of present results with those of Bache et al. [43] and Kit et al. [36] 

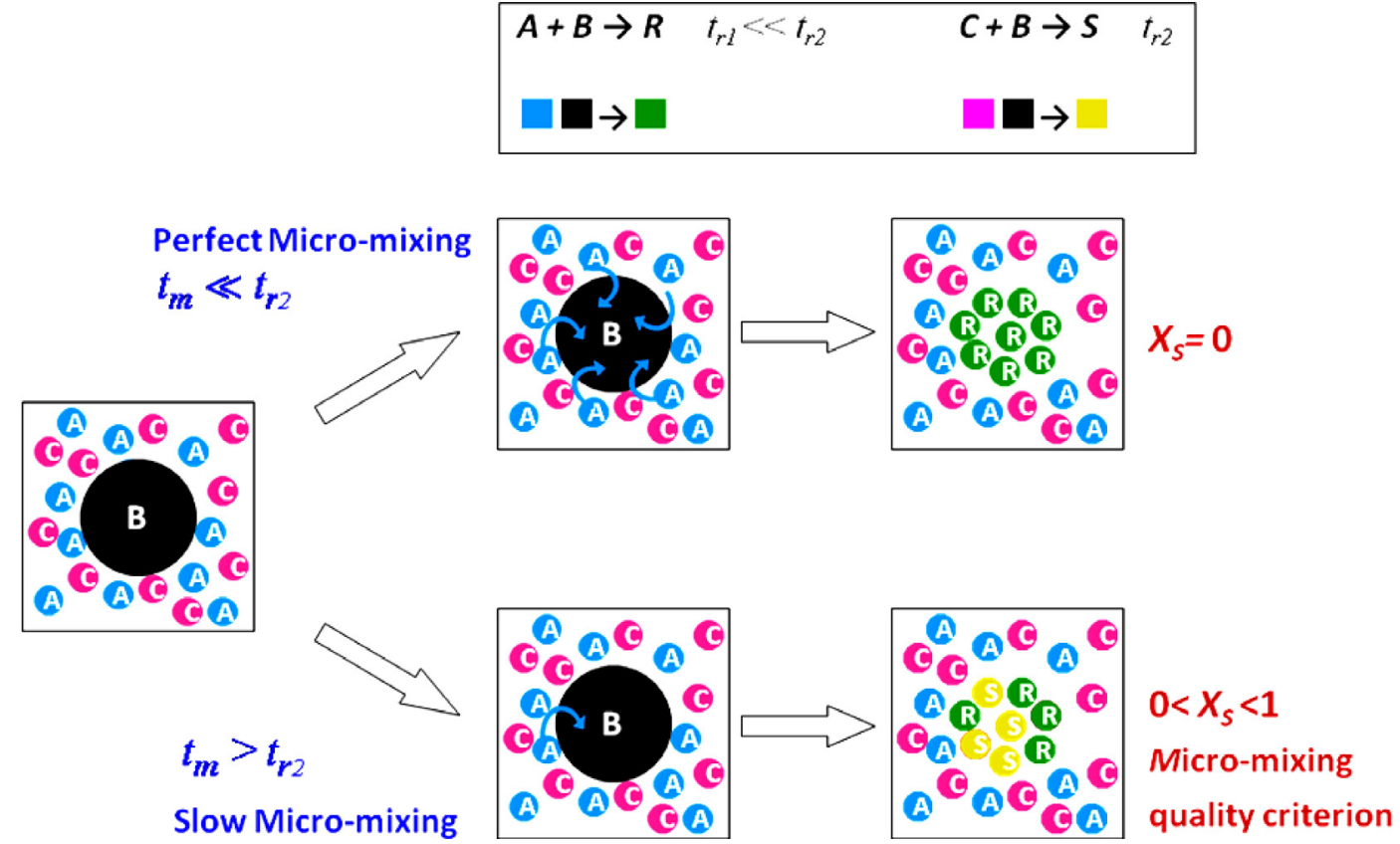

Fig. 11. Principle of micro-mixing process with two chemical reactions of parallel-competitive type (adapted from Habchi et al. [25]).

iodide/iodate reaction as a chemical probe for micro-mixing in a batch chemical reactor. A volume $V_{i n j}$ of acid $\left(\mathrm{H}^{+}\right)$is injected into a tank of volume $V_{\text {tank }}$ composed of a globally perfectly mixed combination of iodate $\left(\mathrm{IO}_{3}^{-}\right)$, iodide $\left(\mathrm{I}^{-}\right)$, called potential iodine, and borate $\left(\mathrm{H}_{2} \mathrm{BO}_{3}^{-}\right)$ions. The main chemical reaction is a quasiinstantaneous neutralization reaction of the borate ions:

$\mathrm{H}_{2} \mathrm{BO}_{3}^{-}+\mathrm{H}^{+} \rightarrow \mathrm{H}_{3} \mathrm{BO}_{3}$

The second chemical reaction, called a Dushman reaction, is a slower redox reaction with kinetic characteristic time $t_{r 2}$ of the order of the micro-mixing time $t_{m}$ :

$5 \mathrm{I}^{-}+\mathrm{IO}_{3}^{-}+6 \mathrm{H}^{+} \rightarrow 3 \mathrm{I}_{2}+3 \mathrm{H}_{2} \mathrm{O}$

The third reaction is the quasi-instantaneous equilibrium of the ions $\left(\mathrm{I}^{-}\right)$and $\left(\mathrm{I}_{3}^{-}\right)$:

$\mathrm{I}^{-}+\mathrm{I}_{2} \rightarrow \mathrm{I}_{3}^{-}$

The kinetics of the three reactions were established by Guichardon et al. [21]. Only the characteristic time of the slowest reaction in Eq. (18) is described here, since it is used for further calculations:

$t_{\mathrm{r} 2}=\frac{\operatorname{Min}\left(\frac{3}{5}\left[\mathrm{I}^{-}\right], 3\left[\mathrm{IO}_{3}^{-}\right], \frac{1}{2}\left[\mathrm{H}^{+}\right]\right)}{r_{2}}$

where the brackets denote the reagent concentration and $r_{2}$ is the rate of the second reaction:

$r_{2}=K_{2}\left[\mathrm{I}^{-}\right]^{2}\left[\mathrm{H}^{+}\right]^{2}\left[\mathrm{IO}_{3}^{-}\right]$

where the constant $K_{2}$ is a function of the ionic strength as given by Guichardon et al. [21].

A local over-concentration of $\mathrm{H}^{+}$produces iodine $\mathrm{I}_{2}$ (Eq. (18)), which itself reacts with iodide $\mathrm{I}^{-}$and yields $\mathrm{I}_{3}^{-}$ions (Eq. (19)). The presence of $\mathrm{I}_{2}$ and $\mathrm{I}_{3}^{-}$is hence the manifestation of a mixing time smaller than the second reaction time and can lead to the segregation index. The segregation index $X_{S}$ is the ratio of acid consumed by the redox reaction (18) on the entire injected acid, defined in a vessel as:
$X_{S}=\frac{Y}{Y_{S T}}$

where the notations $Y$ and $Y_{S T}$ are those chosen by Fournier et al. [19], index ST is used in the case of worst mixing, named state of total segregation:

$Y=\frac{2 V_{\operatorname{tank}}\left(\left[\mathrm{I}_{2}\right]+\left[\mathrm{I}_{3}^{-}\right]\right)}{V_{\text {inj }}\left[\mathrm{H}^{+}\right]_{0}}$

and

$Y_{S T}=\frac{6\left[\mathrm{IO}_{3}^{-}\right]_{0}}{\left[\mathrm{H}_{2} \mathrm{BO}_{3}^{-}\right]_{0}+6\left[\mathrm{IO}_{3}^{-}\right]_{0}}$

The mass balance, the growth of the uniform mixing zone of concentration $c_{i}$, is given by the temporal differential equation:

$\frac{d c_{i}}{d t}=\frac{1}{t_{m}}\left(\left\langle c_{i}\right\rangle-c_{i}\right)+R_{i}$

where $\left\langle c_{i}\right\rangle$ is the mean concentration value in the environment before mixing is completed and $R_{i}$ is the rate of formation of substance $i$ by a chemical reaction. The initial conditions are known (defined by the concentrations), and the $X_{S}$ is computed over a wide range of $t_{m}$ values. The right $t_{m}$ is obtained by identification with the experimental value of $X_{S}$.

\subsection{Experimental procedure}

For measuring $\left[\mathrm{I}_{3}^{-}\right]$and thus the segregation index $X_{S}$, a sample of liquid is transferred to a quartz tank for spectrophotometric analysis after homogenization of the volume. The absorption coefficient $D_{\lambda}$ is measured for a wavelength of $353 \mathrm{~nm}$, which is the absorption peak of the $\mathrm{I}_{3}^{-}$and discriminates the other chemical species. The $\left[\mathrm{I}_{3}^{-}\right]$concentration is calculated by the Beer-Lambert law:

$\left[\mathrm{I}_{3}^{-}\right]=\frac{D_{\lambda}}{\varepsilon_{\lambda} l}$

with $l$ the optical length given by the width of the quartz tank $(10 \mathrm{~mm})$ and $\varepsilon_{\lambda}$ the molar extinction coefficient of $\mathrm{I}_{3}^{-}$ions. This extinction coefficient was measured with an identical spectropho- 
tometer at $2606 \mathrm{~m}^{2} \mathrm{~mol}^{-1}$ by Ferrouillat et al. [48] and $2590 \mathrm{~m}^{2}$ $\mathrm{mol}^{-1}$ by Fournier et al. [19]. By comparison, the extinction coefficient of iodine at $353 \mathrm{~nm}$ is equal to $2 \mathrm{~m}^{2} \mathrm{~mol}^{-1}$ [49].

Once the adjustments are made, the experiment starts by setting the electric motor at a given oscillation frequency. After waiting several minutes to reach steady state, the sulphuric acid is injected. For experimental reliability, the injection time must be long compared with the macro-mixing and meso-mixing time, and so that the injection flow is ensured not to perturb the local turbulence, by assessing the independence of $X_{S}$ with respect to the flow-rate. Visual monitoring checks for the absence of structures resulting from jet turbulence. The injected volume must be as small as possible to ensure local measurements; the volumes are respectively $5 \mathrm{~mL}$ and $10 \mathrm{~mL}$ for Run 5 and Run 6 during $600 \mathrm{~s}$, which provides smaller flow-rates than Fournier et al. [19], around $1 \mathrm{~mL} / \mathrm{min}$. The run identifications are those used by Fournier et al. [19], details of which are reported in Table 2. When the injection is completed, the agitation frequency is then regulated at its maximum for a duration of one minute in order to homogenize the whole tank, and then the electric motor is stopped. A sample can now be taken by a $50 \mathrm{~mL}$ syringe for analysis.

For each operating condition (frequency, height and stroke of grid), three successive injections of acid allow three measurements of $X_{S}$ for the same initial solution. The first measurement of $X_{S}$ is done knowing the initial concentrations. For the second injection, knowing the acid quantity that participated in the acid-base reaction and with the redox reaction during the first injection, all concentrations are re-initialized to calculate new $X_{S}$. This process is repeated until the absorption measurement of $\mathrm{I}_{3}^{-}$becomes impossible in the spectrophotometer.

A matter balance from the stoichiometric Eqs. (17)-(19) is then used to calculate $X_{S}$. Finally, $X_{S}$ is determined with a relative precision of $6 \%$.

\subsection{Segregation index measurements}

Three series of measurements of the segregation index $X_{S}$ are carried out for the different reagents concentrations shown in Table 2: first, a strong iodine concentration coupled with a weak acid concentration (Runs 5 and 6), and second, a weak iodine concentration coupled with a strong acid concentration (Run 2). The two first runs are the least economical solution because of the larger quantity of iodized reagents used.

Experimental measurements of the segregation index $X_{S}$ obtained in grid turbulence in this study (homogeneous case) are summarized in Fig. 12. Following the acid concentration, the level of $X_{S}$ is different for the same hydrodynamic conditions and thus is not an intrinsic measurement of micro-mixing. Acid concentration $\left[\mathrm{H}^{+}\right]=0.08 \mathrm{~mol} / \mathrm{L}$ gives a segregation index greater than those obtained at $\left[\mathrm{H}^{+}\right]=0.04 \mathrm{~mol} / \mathrm{L}$, as confirmed by the results of Fournier et al. (1996): the measured value of $X_{S}$ is directly influenced by the acid concentration.

Fig. 12 also highlights a linear decrease of $X_{S}$ with the Taylor Reynolds number $\operatorname{Re}_{\lambda}$ as observed in Fournier et al. [19]. The slopes of the segregation index as a function of $R e_{\lambda}$ are almost the same

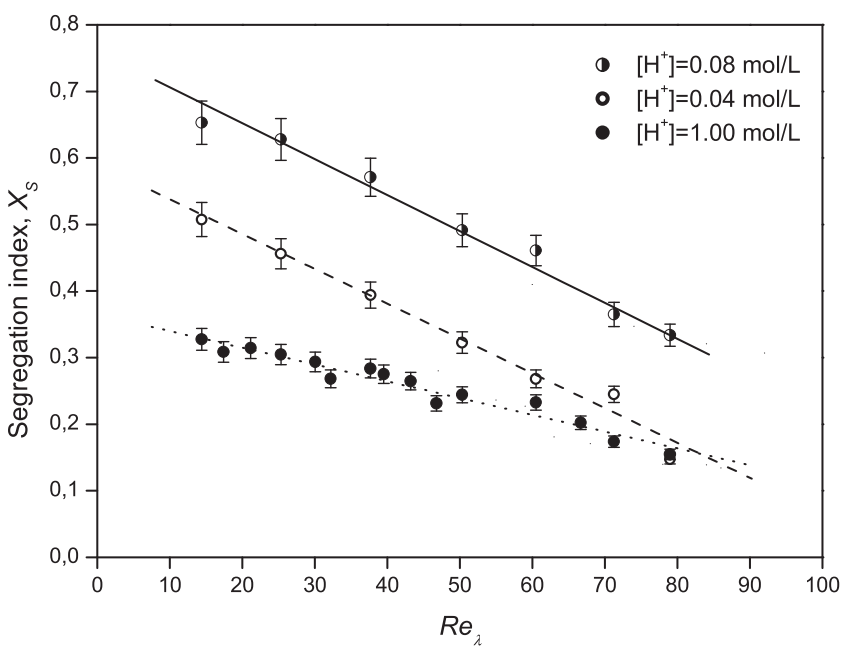

Fig. 12. Segregation index $X_{S}$ according to Reynolds number based on Taylor microscale $R e_{\lambda}$.

for low acid concentrations $\left[\mathrm{H}^{+}\right]=0.08 \mathrm{~mol} / \mathrm{L}$ and $\left[\mathrm{H}^{+}\right]=0.04 \mathrm{~mol} / \mathrm{L}$. For these acid concentrations, potential iodine is the same, $\left[I_{2}\right]_{\text {potential }}=0.07 \mathrm{~mol} / \mathrm{L}$. On the other hand, for the results obtained with $\left[\mathrm{H}^{+}\right]=1.00 \mathrm{~mol} / \mathrm{L}$, the slope is half as large: in this case, potential iodine is ten times lower, $\left[\mathrm{I}_{2}\right]_{\text {potential }}=0.007 \mathrm{~mol} / \mathrm{L}$. Thus the greater the iodine concentration, the greater the slope, and $X_{S}$ measurement is then more sensitive to small turbulence variations.

\section{Micro-mixing time}

In order to quantify the micro-mixing independently of the chemical reaction, it must be modeled in a simple framework that can relate a measured segregation rate $X_{S}$ to a micro-mixing time $t_{m}$. This time $t_{m}$ must depend only on the turbulence occurring at the position where the reactant $B$ is injected. Interest is thus growing in modeling the micro-mixing to establish the link between the progress of the chemical reaction and the micromixing process. These micro-mixing models quantify the share of the reagents perfectly mixed on a molecular scale - and thus available for the reaction - by fitting a micro-mixing time associated to the scalar exchange between a perfectly mixed fluid fraction and the surrounding fluid in 0 -dimensional flows. Here two deterministic models are described and compared: IEM and EDD models.

\subsection{IEM model}

The IEM (Interaction Exchange with the Mean) model is a "twoenvironment" model developed on the basis of the coalescence redispersion model [50], which is a stochastic mixing model. The authors globalize the coalescence re-dispersion model to get a deterministic formulation that requires less computer capacity. The IEM model was validated for a chemical reaction in a dispersed phase in a reactor and extended to a single-phase flow. The authors

Table 2

Concentrations used for the chemical probe.

\begin{tabular}{|c|c|c|c|c|}
\hline Run $^{*}$ & & {$\left[\mathrm{H}^{+}\right]_{0}(\mathrm{~mol} / \mathrm{L})$} & {$\left[\mathrm{I}_{2}\right]_{\text {potential } 0}(\mathrm{~mol} / \mathrm{L})$} & {$\left[\mathrm{H}_{2} \mathrm{BO}_{3}^{-}\right]_{0}(\mathrm{~mol} / \mathrm{L})$} \\
\hline Fournier et al. [19] & Run 5 & 0.08 & $7 \times 10^{-2}$ & $5.66 \times 10^{-2}$ \\
\hline & Run 6 & 0.04 & $7 \times 10^{-2}$ & $5.66 \times 10^{-2}$ \\
\hline Guichardon et al. [20] & Run 2 & 1.00 & $7 \times 10^{-3}$ & $9.09 \times 10^{-2}$ \\
\hline
\end{tabular}

${ }^{*}$ The runs identification used by Fournier et al. [19] and Guichardon et al. [20] are preserved for more clearness. 
underline the difficulty of studying the micro-mixing independently from macro-mixing, and thus this model can include both macro- and micro-mixing, according to the objectives.

In this model it is assumed that at a given instant one chemical species is concentrated in the $\beta$ environment and mass transfer takes place with the chemical species in the $\alpha$ environment (Fig. 13). The respective volumes of $\alpha$ and $\beta$ are constant over time. The intensity of the exchange is scaled by an exchange coefficient $k_{m}$ estimated from a characteristic frequency for isotropic turbulent flows [51] corresponding to the characteristic time of the turbulent cascade:

$k_{m}=\frac{1}{t_{m I E M}}=\frac{1}{C_{1}} \frac{\varepsilon}{k}$

where $C_{1}$ is a constant of order 0.5 and $t_{m \text { IEM }}$ is the micro-mixing time in the IEM model:

$t_{m I E M}=C_{1} \frac{k}{\varepsilon}$

The micro-mixing time $t_{m \text { IEM }}$ was experimentally confirmed in chemical reactors by several authors [52,53], and the IEM model was coupled with information from residence time distribution. This time-scale is generally used with two assumptions. First, the cascade of turbulent kinetic energy produced at large scales is in equilibrium, with its dissipation occurring around the Kolmogorov scale. Second, the cascade time (Corssin time scale) defined by

$t_{t}=\frac{k}{\varepsilon}$

is proportional to the scalar variance dissipation time $t_{\theta}$ :

$t_{\theta}=R t_{t}$

with $R$ a constant. Several authors have shown that $R$ in the range 0.6-3.1 is not a universal constant and depends on the scalar scales [54]. The concentration evolution for the $\alpha$ and $\beta$ environments is given by:

$\frac{d c_{\alpha \text { or } \beta}}{d t}=k_{m}\left(\langle c\rangle-c_{\alpha \text { or } \beta}\right)$

where $c_{\alpha \text { or } \beta}$ is the scalar concentration in the $\alpha$ or $\beta$ environment and $\langle c\rangle$ the mean concentration of the scalar in the whole zone, given by:

$\langle c\rangle=\frac{V_{\alpha} c_{\alpha}+V_{\beta} c_{\beta}}{V_{\alpha}+V_{\beta}}$

with $V_{\alpha}$ the volume of $\alpha$ and $V_{\beta}$ the volume of $\beta$. The advantage of the IEM model lies in its simplicity. Its principal disadvantage is the lack of physical links with the mixing mechanism at small scales. Moreover, its micro-mixing time is not clearly (experimentally) defined in relation to the turbulence properties.

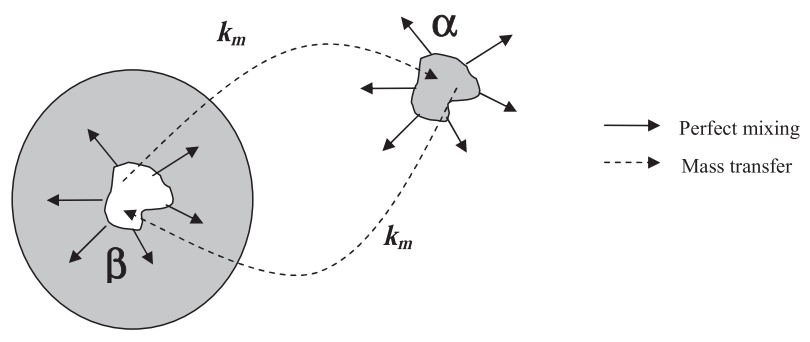

Fig. 13. IEM model.

\subsection{EDD model}

The EDD (Engulfment, Deformation and Diffusion) model is a "two-environment" model: blobs of one fluid environment erode to the benefit of the second environment by fluid breakage and deformation; the final mixing occurs by molecular diffusion. Bałdyga and Bourne [4] showed that in the first step, the blobs break up by turbulent cascade in the inertial zone and are deformed in the second step by fine-scale turbulence; the third step is laminar stretching at the Kolmogorov scale and the fourth is the engulfment process. The restrictive step for molecular mixing is this engulfment process: the engulfment mechanism reduces the non-homogeneity scale by incorporation of the surrounding fluid $A$ in the volume $B$ by the most hydrodynamically active vortices (Fig. 14). These vortices produce a stretched and folded structure of $A$ and $B$ that reduces the scale of $A$ and $B$ (striation thicknesses) until the Batchelor scale is reached; this permits the molecular diffusion mechanism to come to action at the interfaces between $A$ and $B$.

During engulfment, the $B$ environment is assumed to be well mixed at all scales, even after the incorporation of a certain volume of $A$. The growth of the volume $V(t)$ of this perfectly mixed environment is given by an equation involving the characteristic growth time $\tau_{w}$ :

$V(t)=V(0) 2^{t / \tau_{w}}$

identified by

$V(t)=V(0) e^{E t}$

to give $E$, the engulfment parameter. The micro-mixing time $t_{m E D D}$ is then given by:

$t_{m E D D}=\frac{1}{E}$

The evolution of the quantity $n$ of scalar in the volume $V(t)$ is given by $q$, the rate of incorporation:

$\frac{d n}{d t}=c_{0} q=\frac{d(c V)}{d t}$

where $c_{0}$ is the concentration of the scalar outside the volume $V(t)$ and $c$ the concentration inside $V(t)$. Defining a first order law for the volume increase:

$q=\frac{d V}{d t}=E V$

These two Eqs. (36) and (37) lead to the kinetic equation for this micro-mixing model:

$\frac{d c}{d t}=E\left(c_{0}-c\right)$

In the EDD model, the external concentration $c_{0}$ is constant over time but the reference volume grows. Thus the mean concentration is given by:

$\langle c\rangle=\frac{V(t) c+\left[V_{t}-V(t)\right] c_{0}}{V_{t}}$

where $V_{t}$ is the total volume.

\subsection{Micro-mixing time results}

Experimental micro-mixing times are computed with a numerical model, by an "inverse method" that is applied to yield the same segregation index $X_{S}$ as the experimental data. The numerical resolution is carried out by integration over time of the set of equations (31) for IEM and (38) for EDD, for each chemical species of the iodide/iodate system. 


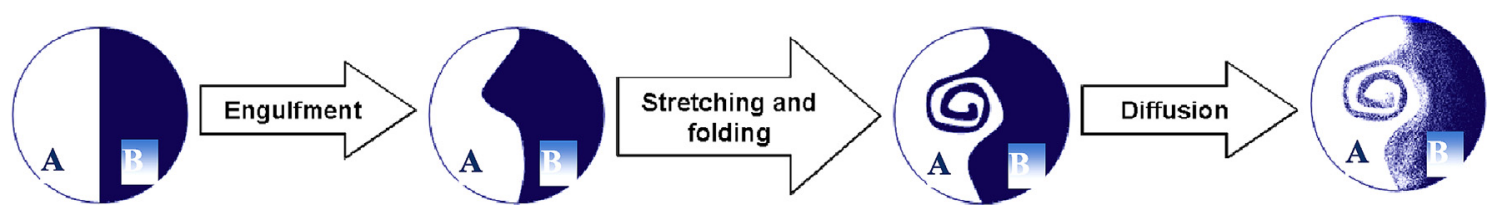

Fig. 14. Schematic engulfment process of the engulfment model.

Fig. 15 shows the computed results of the IEM micro-mixing time $t_{m I E M}$ plotted in log-log scale versus the turbulent dissipation rate $\varepsilon$ obtained by LDA. It is first observed that this model is highly sensitive to the acid concentration. On the one hand, no solution can be attained with $\left[\mathrm{H}^{+}\right]=1.00 \mathrm{~mol} / \mathrm{L}$. On the other, for $\left[\mathrm{H}^{+}\right]=0.08 \mathrm{~mol} / \mathrm{L}$ one notes that $t_{\text {IEM }}$ exceeds $1 \mathrm{~s}$ for a turbulent energy dissipation rate $\varepsilon$ in the range studied $\left[5 \times 10^{-4}-10^{-1}\right]$ $\mathrm{m}^{2} \mathrm{~s}^{-3}$, much more than Baldyga's model predicts, and moreover the trend is not consistent with the $-1 / 2$ law power of $\varepsilon$ by that same model.

$t_{m I E M}=C_{I E M}\left(\frac{v}{\varepsilon}\right)^{1 / 2}$

Unfortunately, the value of the constant $C_{I E M}=32.4$ is much higher than 17.24 given by Bałdyga and Bourne [4] in Eq. (1); even in this case the error in the micro-mixing time is about $88 \%$.

Fig. 16 shows the results of EDD micro-mixing time $t_{m E D D}$ in a similar representation. It is first observed that the micro-mixing times appear rather independent of the operating conditions, even with an order-of-magnitude difference in the acid concentrations. In particular, the results are consistent for the more intense turbulence, that is for $\varepsilon>10^{-2} \mathrm{~m}^{2} \mathrm{~s}^{-3}$ (i.e. Reynolds number $R e_{\lambda}>35$ ). Moreover, the micro-mixing time $t_{m E D D}$ seems to be well scaled by the Kolmogorov time-scale with the $-1 / 2$ log-slope and has values much closer to Baldyga's model. By fitting the experimental results with the expression

$t_{m E D D}=C_{E D D}\left(\frac{v}{\varepsilon}\right)^{1 / 2}$

it is found that $C_{E D D}=12.7$ for the whole set of experiments, which is in quite better agreement with the value of 17.24 reported in Eq. (1). In greater detail, the results for the runs $\left[\mathrm{H}^{+}\right]=0.04 \mathrm{~mol} / \mathrm{L}$ and $0.08 \mathrm{~mol} / \mathrm{L}$ are generally more satisfactory for the whole range of $\varepsilon$. For $\left[\mathrm{H}^{+}\right]=1.00 \mathrm{~mol} / \mathrm{L}$, the results for

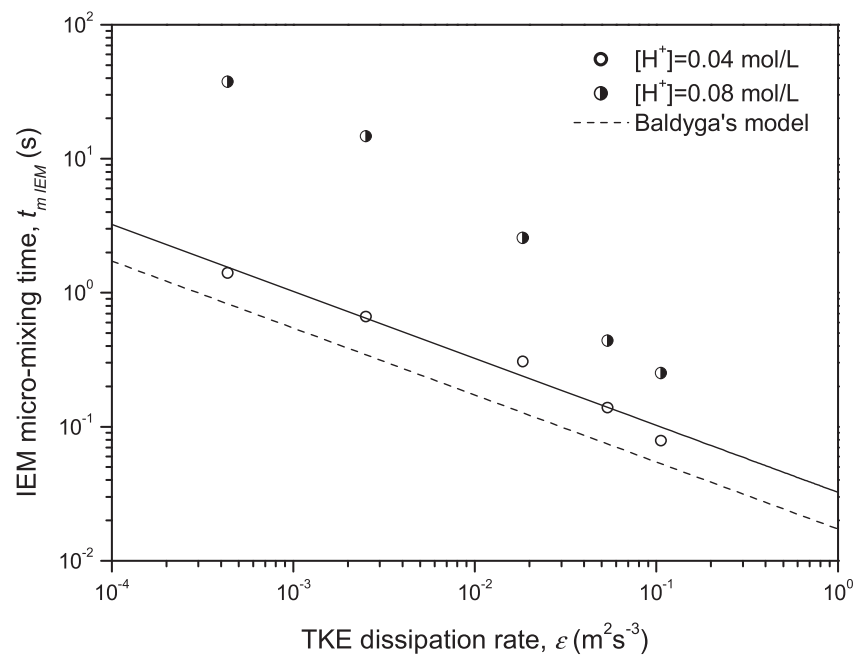

Fig. 15. Experimental micro-mixing time $t_{\text {mIEM }}$ using IEM model versus the dissipation rate $\varepsilon$.

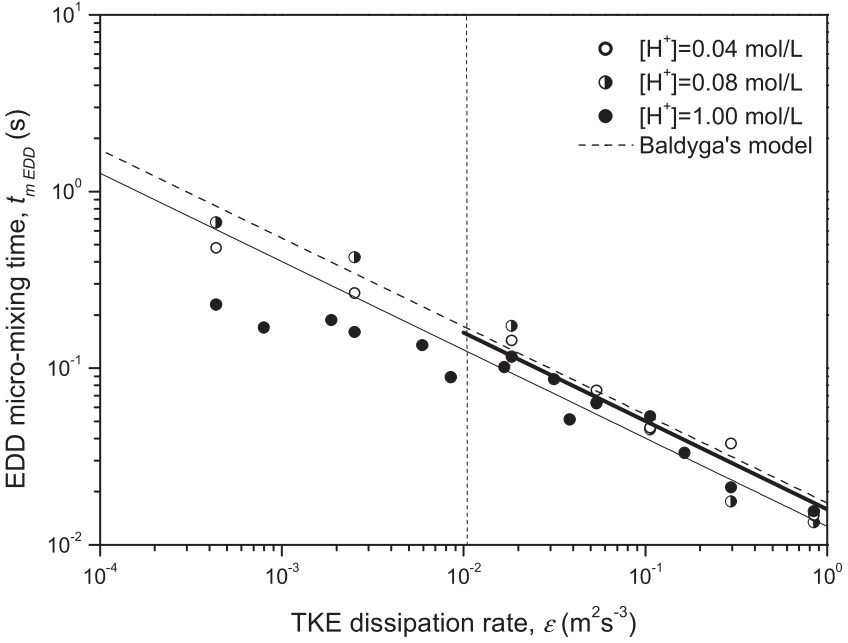

Fig. 16. Experimental micro-mixing time $t_{m E D D}$ using EDD model versus the dissipation rate $\varepsilon$.

$t_{m E D D}$ are actually consistent with $\varepsilon>10^{-2} \mathrm{~m}^{2} \mathrm{~s}^{-3}$ (i.e. $R e_{\lambda}>35$ ), but for $\varepsilon<10^{-2} \mathrm{~m}^{2} \mathrm{~s}^{-3}$ (i.e. $R e_{\lambda}<35$ ) $t_{m E D D}$ seems to move away from the prediction. For low turbulence levels, some limitations in accuracy appear for the method, suggesting that the choice of operating concentration is important. By eliminating this fraction of the experiments, i.e. considering only the results for $\varepsilon>10^{-2}$ $\mathrm{m}^{2} \mathrm{~s}^{-3}$, the constant of Eq. (41) is $C_{E D D}=15.9$ (bold line in Fig. 16).

\section{Discussion}

The IEM model is very sensitive to the change in species concentration. For the higher acid concentration, i.e. $\left[\mathrm{H}^{+}\right]=1.00 \mathrm{~mol} / \mathrm{L}$, the IEM model failed to reproduce the level of $X_{S}$. In this case, the simulated $X_{S}$ is below the experimental value, implying that the IEM model underestimates iodine production. Various features may explain this result.

Consider Eq. (42) representing the molar flux of acid that goes from a $\beta$ environment where acid is predominant to an $\alpha$ environment where borate ions predominate:

Acid flux $_{(\beta) \rightarrow(\alpha)}=\frac{\Delta t}{t_{m}}\left[\mathrm{H}^{+}\right] \frac{V_{\text {tank }} V_{i n j}}{V_{\text {tank }}+V_{i n j}}$

This flux increases with acid concentration, and the acid transferred in the $\alpha$ environment fully reacts with the borate ions. Thus the higher this flux, the less can acid reacts with iodide and iodate to create iodine. For high acid concentration, the IEM model favors the reaction (15). Moreover, the volumes of $\alpha$ and $\beta$ were chosen in order to represent the acid volume and tank volume. Thus, the interaction between the fraction of fluid where the chemical reactions occur and the fresh fluid is neglected, so that the IEM model fails to describe the mixing process properly. For these reasons, this model cannot be used for local simulation of micro-mixing and must be modified to better account for the local aspect of the injection and limit its sensitivity to the concentrations. 
A contrario, the construction of the EDD model can explain its robustness. Actually, EDD model "considers" a medium of acid in which the micro-mixing mechanism incorporates a fraction of the reagents of the surrounding medium, the buffer solution and potential iodine. The acid is always dominating in a perfectly agitated medium, and thus the micro-mixing, which controls the quantity of incorporated matter, readily determines the quantity of iodine formed. It thus seems logical that the micro-mixing phenomenon primarily determines the segregation index $X_{S}$.

This benchmarking shows that EDD model is more adapted to chemical probe analysis, unless the choice of the concentrations is an issue. Moreover, this model consumes less machine time and is simpler to implement since it requires few parameters.

\section{Conclusions}

The chemical probe is a series of reactions whose kinetics is of the order of the micro-mixing rate, which hence becomes indirectly observable. The general configuration in which the chemical probe method was used, like stirred vessels or static mixers, do not let us assess the validity of micro-mixing models since the conditions are far from HIT turbulence, which is the grounding assumption of these models. This induces bias in the results because of advection and turbulent field gradients.

To the best of our knowledge, no earlier work reports on micromixing measurements in a turbulence approaching isotropy and homogeneity with no mean flow. In the present work, chemical probe measurements are carried out in a stirred vessel with a pair of oscillating grids providing a satisfying HIT, for different operating conditions (stroke and frequency) and reagent concentrations. The IEM and EDD micro-mixing models can hence be tested in close to ideal conditions and their results can be compared with the theory.

The IEM model appears to be highly sensitive to the acid concentration and cannot be used with confidence for local measurement for intrinsic characterization of micro-mixing, while EDD model seems to deal consistently with the chemical probe data. The latter model can be taken as a reference among the deterministic micro-mixing models in further experiments. Future work will focus on investigation of the appropriate concentrations and on the deviation of the chemical probe in non-ideal conditions of the complex geometries in real chemical reactors.

\section{Acknowledgements}

Authors would like to acknowledge C. Durandal for his implication in the experimental work.

\section{References}

[1] A. Ghanem, T. Lemenand, D. Della Valle, H. Peerhossaini, Static mixers: mechanisms, applications, and characterization methods - a review, Chem. Eng. Res. Des. 92 (2014) 205-228.

[2] C. Habchi, T. Lemenand, D. Della Valle, M. Khaled, A. Elmarakbi, H. Peerhossaini, Mixing assessment by chemical probe, J. Ind. Eng. Chem. 20 (2014) 1411-1420.

[3] H.J. Yang, G.W. Chu, Y. Xiang, J.F. Chen, Characterization of micromixing efficiency in rotating packed beds by chemical methods, Chem. Eng. J. 121 (2006) 147-152.

[4] J. Baldyga, J.R. Bourne, Turbulent Mixing and Chemical Reactions, Wiley, 1999.

[5] S.W. Jones, O.M. Thomas, H. Aref, Chaotic advection by laminar flow in a twisted pipe, J. Fluid Mech. 29 (1989) 335-357.

[6] C. Habchi, S. Ouarets, T. Lemenand, D. Della Valle, J. Bellettre, H. Peerhossaini, Influence of viscosity ratio on droplets formation in a chaotic advection flow, Int. J. Chem. React. Eng. 7 (2009).

[7] F.J. Muzzio, C. Meneveau, P.D. Swanson, Scaling and multifractal properties of mixing in chaotic flows, Phys. Fluids A Fluid (1992) 1439-1456.

[8] R.O. Fox, Computational Models for Turbulent Reacting Flows, Cambridge University Press, 2003.
[9] J. Baldyga, R. Pohorecki, Turbulent micromixing in chemical reactors - a review, Chem. Eng. J. 58 (1995) 183-195.

[10] G.K. Batchelor, The Theory of Homogeneous Turbulence, Cambridge University Press, 1953.

[11] J. Baldyga, W. Podgórska, R. Pohorecki, Mixing-precipitation model with application to double feed semibatch precipitation, Chem. Eng. Sci. 50 (1995) 1281-1300.

[12] L. Falk, E. Schaer, A PDF modelling of precipitation reactors, Chem. Eng. Sci. 56 (2001) 2445-2457.

[13] R. Pohorecki, J. Baldyga, The effects of micromixing and the manner of reactor feeding on precipitation in stirred tank reactors, Chem. Eng. Sci. 43 (1988) 1949-1954.

[14] G. Tosun, A mathematical model of mixing and polymerization in a semibatch stirred-tank reactor, AIChE J. 38 (1992) 425-437.

[15] J. Aubin, M. Ferrando, V. Jiricny, Current methods for characterising mixing and flow in microchannels, Chem. Eng. Sci. 65 (2010) 2065-2093.

[16] N. Kockmann, T. Kiefer, M. Engler, P. Woias, Silicon microstructures for high throughput mixing devices, Microfluid. Nanofluid. 2 (2006) 327-335.

[17] V. Hessel, S. Hardt, H. Löwe, F. Schönfeld, Laminar mixing in different interdigital micromixers: I. Experimental characterization, AIChE J. 49 (2003) 566-577.

[18] J. Villermaux (Ed.), Génie de la réaction chimique: conception et fonctionnement des réacteurs, Technique et Documentation, Lavoisier, Paris, 1993.

[19] M.-C. Fournier, L. Falk, J. Villermaux, A new parallel competing reaction system for assessing micromixing efficiency-experimental approach, Chem. Eng. Sci. 51 (1996) 5053-5064.

[20] P. Guichardon, L. Falk, Characterisation of micromixing efficiency by the iodide - iodate reaction system. Part I : experimental procedure, Chem. Eng. Sci. 55 (2000) 4233-4243.

[21] P. Guichardon, L. Falk, J. Villermaux, Characterization of micromixing efficiency by the iodide-iodate reaction system. Part II: kinetic study, Chem. Eng. Sci. 55 (2000) 4245-4253.

[22] J.R. Bourne, M. Lips, Micromixing in grid-generated turbulence: theoretical analysis and experimental study, Chem. Eng. J. 47 (1991) 155-162.

[23] M. Assirelli, W. Bujalski, A. Eaglesham, A.W. Nienow, Study of micromixing in a stirred tank using a rushton turbine, Chem. Eng. Res. Des. 80 (2002) 855-863.

[24] S. Ferrouillat, P. Tochon, C. Garnier, H. Peerhossaini, Intensification of heattransfer and mixing in multifunctional heat exchangers by artificially generated streamwise vorticity, Appl. Therm. Eng. 26 (2006) 1820-1829.

[25] C. Habchi, D. Della Valle, T. Lemenand, Z. Anxionnaz, P. Tochon, M. Cabassud, et al., A new adaptive procedure for using chemical probes to characterize mixing, Chem. Eng. Sci. 66 (2011) 3540-3550.

[26] J. Baldyga, J.R. Bourne, Simplification of micromixing calculations. I. Derivation and application of new model, Chem. Eng. J. 42 (1989) 83-92.

[27] J. Baldyga, J.R. Bourne, Comparison of the engulfment and the interaction-byexchange- with-the-mean micromixing models, Chem. Eng. J. 45 (1990) 2531.

[28] J. Baldyga, A. Rozeń, F. Mostert, A model of laminar micromixing with application to parallel chemical reactions, Chem. Eng. J. 69 (1998) 7-20.

[29] C. Durandal, T. Lemenand, D. Della Valle, H. Peerhossaini, A chemical probe for characterizing turbulent micromixing, in: ASME Fluids Eng. Div. Summer Meet. Symp. Macro- Micromixing Single Phase Fluids, ASME, Miami, 2006, pp. 1091-1099.

[30] S.B. Pope, Turbulent Flows, Cambridge University Press, 2000.

[31] S.S. Shy, C.Y. Tang, S.Y. Fann, A nearly isotropic turbulence generated by a pair of vibrating grids, Exp. Therm. Fluid Sci. 14 (1997) 251-262.

[32] K. Abou Hweij, F. Azizi, Hydrodynamics and residence time distribution of liquid flow in tubular reactors equipped with screen-type static mixers, Chem. Eng. J. 279 (2015) 948-963.

[33] Q. Zhou, N.S. Cheng, Experimental investigation of single particle settling in turbulence generated by oscillating grid, Chem. Eng. J. 149 (2009) 289-300.

[34] H.J.S. Fernando, I.P.D. De Silva, Note on secondary flows in oscillating-grid, mixing-box experiments, Phys. Fluids A Fluid Dyn. 5 (1993) 1849.

[35] I.P.D. De Silva, H.J.S. Fernando, Oscillating grids as a source of nearly isotropic turbulence, Phys. Fluids 6 (1994) 2455.

[36] E.L.G. Kit, E.J. Strang, H.J.S. Fernando, Measurement of turbulence near shearfree density interfaces, J. Fluid Mech. 334 (1997) 293-314.

[37] E. Villermaux, B. Sixou, Y. Gagne, Intense vortical structures in grid-generated turbulence, Phys. Fluids 7 (1995) 2008.

[38] Y. Zellouf, P. Dupont, H. Peerhossaini, Heat and mass fluxes across density interfaces in a grid-generated turbulence, Int. J. Heat Mass Transf. 48 (2005) 3722-3735.

[39] K.C. Kim, R.J. Adrian, Very large-scale motion in the outer layer, Phys. Fluids 11 (1999) 417.

[40] T. Lemenand, P. Dupont, D. Della Valle, H. Peerhossaini, Turbulent mixing of two immiscible fluids, J. Fluids Eng. 127 (2005) 1132.

[41] A. Host-Madsen, C. Caspersen, The limitations in high frequency turbulence spectrum estimation using the laser Doppler anemometer, in: Seventh Int. Symp. Appl. Laser Tech. to Fluid Mech., Lisbon, 1994.

[42] K.R. Sreenivasan, On the scaling of the turbulence energy dissipation rate, Phys. Fluids 27 (1984) 1048-1051.

[43] D.H. Bache, E. Rasool, Measurement of the rate of energy dissipation around an oscillating grid by an energy balance approach, Chem. Eng. J. Biochem. Eng. J. 63 (1996) 105-115. 
[44] H. Mohand Kaci, T. Lemenand, D. Della Valle, H. Peerhossaini, Effects of embedded streamwise vorticity on turbulent mixing, Chem. Eng. Process. Process Intensif. 48 (2009) 1459-1476.

[45] H. Mohand Kaci, C. Habchi, T. Lemenand, D. Della Valle, H. Peerhossaini, Flow structure and heat transfer induced by embedded vorticity, Int. J. Heat Mass Transf. 53 (2010) 3575-3584.

[46] W. Jiao, Y. Liu, G. Qi, A new impinging stream-rotating packed bed reactor for improvement of micromixing iodide and iodate, Chem. Eng. J. 157 (2010) 168 173.

[47] M. Bertrand, N. Lamarque, O. Lebaigue, E. Plasari, F. Ducros, Micromixing characterisation in rapid mixing devices by chemical methods and LES modelling, Chem. Eng. J. 283 (2016) 462-475.

[48] S. Ferrouillat, P. Tochon, H. Peerhossaini, Micromixing enhancement by turbulence: application to multifunctional heat exchangers, Chem. Eng. Process. Process Intensif. 45 (2006) 633-640.
[49] T.L. Allen, R.M. Keefer, The formation of hypoiodous acid and hydrated iodine cation by the hydrolysis of iodine, J. Am. Chem. Soc. 77 (1955) 2957-2960.

[50] R.L. Curl, Dispersed phase mixing: I. Theory and effects in simple reactors, AIChE J. 9 (1963) 175-181.

[51] S.B. Pope, PDF methods for turbulent reactive flows, Prog. Energy Combust. Sci. 11 (1985) 119-192.

[52] X. Guo, Y. Fan, L. Luo, Mixing performance assessment of a multi-channel mini heat exchanger reactor with arborescent distributor and collector, Chem. Eng. J. 227 (2013) 116-127.

[53] M. Kashid, A. Renken, L. Kiwi-Minsker, Mixing efficiency and energy consumption for five generic microchannel designs, Chem. Eng. J. 167 (2011) 436-443.

[54] M. Gonzalez, A. Fall, The approach to self-preservation of scalar fluctuations decay in isotropic turbulence, Phys. Fluids 10 (1998) 654 